\title{
CARGA DE LA PRUEBA Y ESTÁNDAR PROBATORIO: LA INFLUENCIA DE LEO ROSENBERG Y KARL HAINZ SCHWAB PARA EL DESARROLLO DEL MODERNO DERECHO PROBATORIO*
}

Hanns PrüttING**

\section{LAS CIENCIAS JURÍDICAS COMO CIENCIA}

¿Qué hacemos nosotros realmente cuando nos dedicamos (ya desde unos 2000 años) a las Ciencias Jurídicas? O preguntado con mayor claridad: ¿Es ello realmente una ciencia?

Es la pregunta probablemente más provocativa de uno de los legendarios juristas Julius Von Kirchmann. Fue acuñada en 1848 ante la Sociedad de Ciencias Jurídicas de Berlín en un discurso bajo el título "Sobre la inutilidad de la jurisprudencia como ciencia". El entonces fiscal en jefe Von Kirchmann observaba una gran diferencia entre la ley natural y el derecho positivo. Éste impone a sus usuarios y quienes deben aplicarlo instrucciones claras, así: "Los abogados se caracterizan por el empleo del derecho positivo para convertirse en gusanos que sólo viven en la madera podrida, apartándose de los sanos, sólo rodeado de enfermos, donde anidar y desarrollarse. Mientras que la ciencia trabaja con lo accidental del sujeto, es incluso aleatoria, pero bastan tres palabras del legislador para

\footnotetext{
* Conferencia pronunciada con motivo del aniversario del fallecimiento de Karl Heinz Schwab. Traducción de Álvaro Pérez-Ragone, Doctor en Derecho Universidad de Colonia; Profesor de Derecho Procesal Civil en la Pontificia Universidad Católica de Valparaíso. Forma parte del Proyecto FONDECYT 11070054 del traductor y de la Beca Alexander Von Humboldt. Acotación del traductor: este aporte es un homenaje a Karl Heinz Schwab, maestro directo e indirecto por sus discípulos de varias generaciones. Colaboración recibida el 16 de abril y aprobada el 23 de abril de 2010.

** Doctor en Derecho y Habilitation en Erlangen; Catedrático de Derecho Procesal Civil, Laboral e Insolvencia (Ordinarius-Universitätsprofessor); Director del Instituto de Derecho Procesal de la Facultad de Derecho de la Universidad de Colonia, Alemania. Correo electrónico del traductor: alvaro. perez@ucv.cl.
} 
convertir bibliotecas enteras de Derecho en basura de papel" ${ }^{1}$ La última frase es la más famosa y citada.

La pregunta sobre el valor de la Jurisprudencia como ciencia nos conduce a la noción general de ciencia. Si se trata en un sentido general la ciencia como el epítome de una era del conocimiento humano, que reúne sistemáticamente, se enseña y transmite como un conjunto de hallazgos que se refieren a un tema e incluyen un contexto explicativo, claramente no parece especialmente difícil poder incluir al Derecho en este contexto. Se vuelve más difícil cuando nos fijamos en la ciencia como un proceso metodológico de la investigación y el conocimiento intersubjetivamente comprensible. Esta opinión se correspondería más a la presentación de Kirchmann que en ese momento se centró principalmente en las ciencias físicas. Cita: “¡Cuánto mejor sería la ley si pudiera, como las ciencias naturales, directamente operar sobre objeto tratado!".

Incluso Rudolf von Ihering (1818 - 1892), en su conferencia inaugural en Viena el 16 de octubre de 1868 vinculó esas teorías y la dependencia del exterior recordando que el Derecho tiene una dependencia de los acontecimientos políticos y las fronteras nacionales, pero también los peligros del positivismo pueden afectar a la Jurisprudencia: "Este es el enemigo mortal del positivismo jurídico, porque puede ver la nave hacia abajo, y tienen que luchar entre ellos, por lo tanto, por la muerte o la vida". Y después de algunos ejemplos históricos, continúa: "La recurrencia de tales condiciones en diversos momentos de la historia del Derecho justamente, y en diferentes condiciones nos enseñan que aquí no se trata de accidentes que vienen de afuera, sino que tienen su razón en la naturaleza misma del Derecho. Ustedes no necesitan la causa del mal, aunque la misma puede detectarse en una parálisis periódica. Y este mal fundamental de la ley, contra el que se debe estar constantemente en guardia so consecuencia de sucumbir de inmediato a su voluntad, llamado positivismo, es el velo de la propia esencia de la ley cuando se la considera como una herramienta sin sentido" ${ }^{2}$.

Lo que es profundamente triste es que esas palabras fueron adoptadas por muchos juristas alemanes de los años entre 1933 a 1945.

Ihering mismo era conciente de la locuaz y evidente imaginación de Julius Von Kirchmann, cuando concluyó: "Si tomo la suma de lo que he dicho antes, como mi llamado a la conciencia científica de la ley en cosas del Derecho, la conciencia desde la filosofía jurídica del derecho como partida, se tienen que explorar las causas últimas del derecho a partir de su origen en la tierra y ámbito

\footnotetext{
${ }^{1}$ Von Kirchmann, Julius, Die Wertlosigkeit der Jurisprudenz als Wissenschaft, Nueva reimpresión, Darmstadt 1956, p. 25.

${ }^{2}$ VON IHERING, Rudolf, Ist die Jurisprudenz eine Wissenschaft?, Clase inaugural 16.10.1868, publicada en la colección Behrends, Göttingen 1998, pp. 52 y ss.
} 
de aplicación. O de acuerdo a la visión de la historia del derecho, lo acompaña al hombre en todos los caminos que ha adoptado con el fin de que paso a paso se tienda a la más alta perfección, del dogma a la práctica en la visualización científica ordenada de todas las experiencias y hechos, que incluyen el estado actual y el punto final de nuestro conocimiento y la cobertura del Derecho en sí mismo"'3.

Desde entonces se ha considerado al conocimiento jurídico como diferente a la ciencia. Por ejemplo, Gustav Radbruch sostuvo que, incluso el defecto de la ley podría estar en su tipicidad, capacidad de generación y su sujeto como ciencia. Así es por de pronto y como la historia de la ciencia, es al menos en este sentido una ciencia más ${ }^{4}$. Arthur Kaufmann ha argumentado en contra, sosteniendo que la cuestión de si realmente es o no una ciencia puede aun quedar abierto a interrogante. Debido a que el concepto de ciencia no es un dogma, se pudo determinar de manera bastante pragmática su uso y noción en particular. Por supuesto, también se requiere de una serie de criterios que deben cumplirse para poder hablar de ciencia. Por ello llamó a la coherencia en el sistema de generación, la racionalidad, la posibilidad de verificación y finalmente el consenso en torno a la ley. Es evidente, por lo menos, que los métodos científicos son diferentes de aquellos con los que puede contar un juez, pero habría algunos lineamientos desarrollados de forma metódica y que han conformado tradiciones científicas independientes ${ }^{5}$. A diferencia de las ciencias naturales con la observación, formación de hipótesis, experimentación y elaboración de teorías-tesis, los métodos de las humanidades se refieren a un sentido de comprensión y de asignación de significado a los fenómenos individuales en el marco histórico, cultural y normativo de las relaciones. La pregunta clásica de la Jurisprudencia vuelve a plantearse hoy nuevamente, pero con otra apariencia. Se trata de la idea del progreso. Así, en los últimos tiempos se señaló que la ley como tal, está desapareciendo ampliamente de los programas de desarrollo de las organizaciones científicas. Desde hace años, por ejemplo no existe un Premio Leibniz en las ciencias jurídicas. La investigación jurídica se lleva a cabo fuera incluso como conocimiento auxiliar a tópicos económicos, cambios sociológicos, ciencias políticas o la investigación histórica. Esto afecta incluso a los que en los últimos años como juristas intentaron incluir investigaciones jurídicas en proyectos de iniciativas de excelencia, por cierto con enorme dificultad para su calificación. Esto reviste especial observación, cuando se intenta acercar las "Ciencias Jurídicas" a las ciencias naturales o conocimientos técnicos. Más recientemente, incluso cuando el Derecho fue

\footnotetext{
${ }^{3}$ VON IHERING, cit. nota n. 2, p. 92.

${ }^{4}$ Radbruch, Rudolf, Rechtsphilosophie, 9a edición, Beck, Múnich, 1983, p. 218.

${ }^{5}$ Kaufmann, Arthur, $J Z, 1992$, p. 300.
} 
objeto del intento de una cierta formalización lógica de la ley para integrar el procesamiento electrónico de datos. La duración del derecho informático y sus intentos en los años 70 del siglo pasado han sido ampliamente observados y discutidos. Reinhold Zippelius ${ }^{6}$ observó la idea y voluntad de supervivencia para poder contar con la información correcta, pero sin poder aun tener lista una semántica precisa, pudiendo lograrse formulaciones legales calculables, pero no como juicios de valor. Cualquier idea como la del que un equipo informático podría reemplazar el juez nos parece hoy en día poco seria.

Así que si en los pequeños tópicos resulta ciertamente difícil que un sujeto pueda lograr una formalización lógica de la ley, remontándose incluso a dudas planteadas en siglos anteriores, puede cuestionarse en qué medida podría seguirse intentando la aplicación del concepto de ley científica al fenómeno jurídico.

\section{EL DERECHO PROBATORIO COMO CONTRAPOSICIÓN}

El derecho probatorio tiene una gran importancia para la práctica jurídica y la aplicación de la ley. En nuestro contexto, el desarrollo científico del derecho probatorio, particularmente la carga de la prueba, también puede considerarse como un buen ejemplo especialmente del trabajo científico y el progreso científico en el Derecho, el que por cierto se dio de una manera realmente impresionante ${ }^{7}$.

Ello en consonancia con el crecimiento y notable desarrollo del Derecho Procesal Civil durante los siglos XVIII y especialmente XIX en Europa. ¿ Fueron quizá el impulso del proceso común con su escrituración y falta de publicidad del examen judicial de la prueba reflejado en reglas fijas o tasadas de valor, luego disipado con la caída del antiguo imperio y las tormentas de las conquistas napoleónicas que sellaron la caída de estos viejos sistemas aun existentes por entonces? Quizá sí, y en especial nuevos modelos de proceso en los Estados desarrollados, en parte inspirados en las leyes francesas sobre procedimiento. En efecto se dio un cambio radical hacia un proceso oral, público, orientado por las partes, con libre apreciación de las pruebas como reflejo de las ideas de reforma de esa época.

El CPO alemán del año 1877 como parte de las Leyes de Justicia del Imperio, significó un cierre provisorio a esta evolución. Ya no era el uso directo o indirecto del secretismo los que primaban sino principios propios del nuevo proceso por audiencias con el juego de la preclusión y el rol de la eventualidad. Así se fue gradualmente reemplazando también la teoría de la prueba legal. La nueva co-

${ }^{6}$ ZipPELIUS, Juristische Methodenlehre, 10. Aufl. 2006, S. $106 \mathrm{ff}$.

${ }^{7}$ Vgl. dazu insb. M. Ahrens, Prozessreform und einheitlicher Zivilprozess, Tübingen, 2007. 
dificación liberal alemana fue convincente y tuvo una importancia fundamental para la unidad jurídica en Alemania y más allá, una fuerte influencia para muchos otros países en el mundo. Y esto aún tiene validez en la actualidad .

Con la libertad del juez para evaluar todas las pruebas un nuevo problema entró a la escena judicial, por cierto imperceptible inicialmente. La libertad en el derecho de la prueba no era y no es ilimitada. ¿Qué clase de libertad tenía entonces el juez en este nuevo sistema de valoración de la prueba? Esta pregunta ya que, al menos, está obligado por la lógica o ley científica. Basta pensar en las cortes estatales en los casos de "Exorcismo" ante tribunales seculares. ¿Cuándo y bajo qué circunstancias, el tribunal puede ser convencido por cualquier ficto? Es especialmente a partir de mediados del siglo XIX que la jurisprudencia se ocupa sobre la controvertida cuestión de cómo relacionar estos temas con la carga de la prueba. ¿Quién debe soportar y qué reglas debieran regir en el supuesto que el juez continúe aún sin prueba suficiente? A estas y otras preguntas similares de derecho de la prueba se dedicaron un gran número de monografías e investigaciones en la segunda mitad del siglo XIX. Con el principio de la oralidad, el proceso por audiencias y el rol central de la prueba en especial, sumado a la eliminación de antiguas restricciones procesales, muchos arribaron a la conclusión de que todas las pruebas debían estar vinculadas a las peticiones de las partes y que, sobre la base de los debates con alegación y prueba celebrados a lo largo del todo proceso, era posible tener por verificada en la decisión la producción o no de una prueba. La carga (subjetiva) de la prueba dirigida a las partes para el desarrollo de su actividad probatoria a partir de la ley y reflejadas en una decisión judicial.

\section{LeO RosenberG y LA teOría de LA CARGA DE LA PRUEBA}

La carga de la prueba como un fenómeno jurídico ya se conocía en el derecho romano, aunque sólo en forma de carga de la prueba en el actual sentido de carga subjetiva de la prueba. Citado a menudo es la máxima de Paulo "ei incumbit probatio, qui negación Dicit, qui no". Del mismo modo, la postulada por Marciano "semper necessitas probandi incumbit illi, qui agit". La distribución de la carga de la prueba inicialmente partía de fórmulas muy específicas y obligatorias que podían guiar la ponderación judicial sin gran dificultad. Más tarde, la carga de la prueba se determinó en general por la decisión judicial. Ahora el criterio de imposición judicial se mantenía en gran parte, en la oscuridad de la discrecionalidad del juez. A lo largo de los siglos y de estudios se puede constatar que los principios jurídicos romanos desarrollaron una teoría

${ }^{8}$ En relación a esta cita ver comp. PRÜTTING, Hanns, Gegenwartsprobleme der Beweislast, Beck, Múnich, 1983, p. 269. 
de la prueba, que dependía de muchos detalles. Fue recién luego de la Ilamada Teoría Negativa (Negativentheorie) que se planteó una distinción entre los hechos positivos y negativos. Paulatina importancia práctica asumió paralelamente la Teoría de las Presunciones (Präsumtionstheorie), que individualizaba los problemas concretos de la prueba con la ayuda de supuestos, que a su vez se basaban fundamentalmente en una conducta individual remota. Finalmente asumiendo la existencia y continuidad de leyes preexistentes, más tarde desarrollaron la Ilamada Teoría de la Continuidad con un importante y significativo impacto de la decisión en la carga concreta de la prueba, siendo la opinión de que ciertos hechos muy excepcionales pueden afectar la distribución de la carga de la prueba. Por último, la llamada Teoría de la posibilidad probatoria (Erwiesenheitstheorie) de Franz Leonhard ${ }^{9}$ fue desarrollada para permitir sustentar la consecuencia jurídica de una norma sobre la base de la demostración de los hechos, no estando condicionada a la existencia de hechos objetivos. Dicho contenido estaba solamente relacionado con el proceso y, por lo tanto, terminaba reduciendo a tres los posibles resultados de una cognición judicial (ya sea probado, desmentido, o confuso) a una simple dicotomía (probados y no probados).

Todas estas teorías de la prueba en la práctica, ampliando la discreción del juez, generaron gran incertidumbre, sobre todo en las partes y la marcada falta de unanimidad en las decisiones judiciales. En 1913, incluso, Gautschi presentó un estudio en cuyas primeras 15 páginas, sostiene la tesis de que la carga de la prueba no debía juzgarse de acuerdo a la ley, sino la libre discreción del juez. Curiosamente, en las restantes 520 páginas con un ejemplo tras otro no llega siquiera a poder afirmar e identificar un sistema como el propuesto.

En esta situación, Leo Rosenberg (1879-1963) por entonces un joven de 21 años en 1900, presenta una decisiva e importante tesis doctoral sobre la carga de la prueba. La que hasta ahora ha merecido 5 ediciones continuadas. Rosenberg insistió en su postura para finalmente imponerse por y en sus publicaciones. En primer lugar, era necesario que, además de la carga subjetiva de rendir la prueba ofrecida a lo que se le suma una carga objetiva (según Rosenberg), sobre lo que se asienta la decisión judicial. Esta carga para la determinación es en realidad un fenómeno central del que derivan la carga de ofrecer y producir la prueba como la carga de la alegación. En segundo lugar, Rosenberg reconoció que la distribución de la carga de la prueba no estaba sujeta a la discreción del juez, de los principios individuales o los resultados de las hipótesis, sino que tenía sus raíces en la ley, en la naturaleza y estructura de la norma jurídica ${ }^{10}$.

\footnotetext{
${ }^{9}$ Leonhard, Franz, Die Beweislast, 2a edición, Duncker \& Humlot, Berlin, 1904, 1926, p. 127.

${ }^{10}$ Por primera vez sostenido por GlASER, Julius en 1883, Handbuch des Strafprozesses, Leipzig, 1883, p. 364, donde distinguió entre carga de la prueba material y formal.
} 
Esta teoría se reconoció como Teoría del Estándar probatorio y ha prevalecido, siguiendo vinculada aún con Rosenberg. Con ella se preparó la idea fundamental de la carga de la prueba por el legislador como un riesgo distribuido y, por tanto, garantizador de la seguridad jurídica. Esta era-como ahora sabemos-un paso fundamental en la dirección correcta y tiene a la muerte de Rosenberg en 1963 una teoría y práctica suficientemente establecida. Las correcciones a este punto de vista se deben a los estudios de Leipold ${ }^{11}$ al importante debate sobre los hechos impeditivos y extintivos de 1966, lo que se ha añadido al sistema para mayor sistematización y sólo pero poco de los principios subyacentes a la teoría estándar han cambiado. Así, podemos ver que la teoría estándar en una forma modificada pero que sigue siendo hoy base para la teoría y la práctica de la carga de la prueba. Rosenberg no pudo seguir discutiendo, sin embargo, el problema de la medida de la prueba sigue siendo actual. Aunque él ha afirmado que hay que distinguir entre la prueba en el sentido estricto (los motivos de la plena convicción del juez) y la presunción, no ha dedicado a esta distinción, ninguna consideración adicional ${ }^{12}$.

El concepto de medida o estándar de la prueba (Beweismaßbegriff) y sus problemas fundamentales surgen y son tratados recién por la doctrina alemana inmediatamente después de la muerte de Leo Rosenberg.

\section{La doctrina de Karl-Heinz Schwab}

Karl-Heinz Schwab, a diferencia de su maestro Leo Rosenberg, no produjo una monografía dedicada a la carga de la prueba. No es atípico que por su humildad, sin embargo, haya impulsado por otras contribuciones el desarrollo de derecho probatorio. Se inicia con la publicación de la última edición de la prueba, el libro de Rosenberg en 1965 (justo después de su muerte). Después, continuará con la adopción del libro de texto o tratado de Rosenberg sobre Derecho Procesal Civil, es conocido desde la décima hasta la decimocuarta edición de la obra en 1986, un total de cinco ediciones continuadas. Él asumirá en el desarrollo de este libro, por una parte, como el guardián de la doctrina y la evolución de Rosenberg, tratando de actualizar la obra con suma prudencia. Por otra parte, no debe pasarse por alto que con cada complemento y la ampliación de una obra de referencia como el libro de texto de Rosenberg / Schwab se ejerció una gran influencia en la teoría y la práctica del proceso civil. Es claro y evidente que por ello tuvo que intervenir en el debate sobre la prueba. Karl-Heinz Schwab tiene un ensayo en el Libro de Homenaje a HJ Bruns, donde

${ }^{11}$ LeIPOLD, Dieter, Beweislastregeln und gesetzliche Vermutungen, Duncker \& Humlot, Berlin, 1966, pp. 17 y ss.

${ }^{12}$ RosenberG, Leo, Lehrbuch des deutschen Zivilprozessrechts, Berlin 1927, § 113 I 2, p. 342. 
presenta una contribución titulada "Die Abkehr moderner Beweislastlehren Von der Normentheorie" (La desviación del moderno derecho probatorio en relación a la Teoría Normativa-Traducción libre) ${ }^{13}$.

El propio título de esta contribución muestra que defiende la teoría estándar de Rosenberg. Esta nueva defensa parecía urgente, ya que en los años 1975 y siguientes una serie de estudios parciales fueron presentados para intentar sustituir la distribución de la carga de la prueba establecida en la ley y transferir a los principios, tales como la probabilidad in abstracto, la probabilidad in concreto, la teoría de los riesgos y otras elaboraciones similares con un diferente punto de vista. Magistralmente Schwab ha demostrado que todos estos principios no son capaces de sustituir la teoría estándar y que por el contrario crean inseguridad jurídica y por consiguiente tienen consecuencias engañosas. De hecho, las investigaciones de los años 70 del siglo pasado fueron en gran medida un intento de construir sobre las ideas originales una vestimenta más moderna, lo que curiosamente ya había sido desarrollado, aplicado y luego superado durante los siglos XVIII y XIX.

Con especial claridad la influencia de Karl-Heinz Schwab, se refleja en la decimocuarta edición del libro de Rosenberg a partir de 1986. Allí, los recientes estudios científicos sobre la teoría de carga de la prueba se desarrollan con claridad y son incorporados en el sistema básico de la teoría estándar de Rosenberg. Por otro lado quedó muy claro que ni una carga de la prueba por la teoría del riesgo y menos el solo juego de la probabilidad brindaban por sí una solución al problema de la carga de la prueba. A diferencia de Rosenberg, Schwab dedica en esta edición y más tarde en una contribución al Libro de Homenaje a Fasching un capítulo autónomo dedicado a la medida o estándar de la prueba ${ }^{14}$.

Su importancia puede ser plenamente reconocida sólo si se tiene en cuenta los extremos que fueron adoptados por la doctrina y el desarrollo de la materia entre 1975 hasta 1985. Se ha sostenido varias veces, al menos en general, que el estándar probatorio en la ley alemana actual se fundaría en la mayor probabilidad. El juez siempre debe estar convencido cuando se argumenta a favor antes que en contra de una afirmación. Estos postulados fueron impulsados inicialmente por esas teorías Ekelöf ${ }^{15}$ y Motsch ${ }^{16}$ y, en definitiva, persiguen negar

\footnotetext{
${ }^{13}$ S für Bruns; Heymanns, Colonia 1978, p. 505.

${ }^{14}$ RosenberG, Leo; Schwab, Karl-Heinz, Zivilprozessrecht, 14ª edición, Bech, Múnich, 1986, § 113 II; SснwAB, Karl Heinz, Festschrift für Fasching, Mainz, Berna,1988, p. 451.

${ }^{15}$ EKELÖF, Per Olof, Beweiswürdigung, Beweislast und Beweis des ersten Anscheins, ZZP 75,1962, p. 289.

${ }^{16} \mathrm{MotsCH}$, Richard, Vom Prozess als Beweis zum Uberwiegungsprinzip, in Gedächtnisschrift für Rödig, Berlin,1978, p. 334; del mismo, Vom rechtsgenügenden Beweis, Duncker \& Humlot, Berlín, 1983, pp. 93 y ss.
} 
la posibilidad, al menos plena y perfecta, sobre la existencia de una decisión sobre la carga de la prueba. Motsch fundamenta su visión sobre la base de las ideas básicas de la prueba en tanto alto grado de probabilidad, de tal manera que sería imposible pensar un supuesto de falta de prueba completa al momento de decidir. A tal grado que carecería incluso de importancia práctica y que en definitiva impondría al juez el tener que aplicar el famoso examen de probabilidad del 50: 50, a fin de convencerse y persuadir en su sentencia sobre su decisión. Motsch arriba finalmente a su tesis sobre la base del examen judicial de las pruebas en un sistema de libre valoración de la prueba que permitiría solucionar cualquier problema probatorio. Una decisión con arreglo a los principios que regulen la prueba atentarían, según él, contra el § 286 ZPO.

Así es que debe agradecerse a Schwab que tales visiones extremas no lograron instalarse en la doctrina jurídica alemana, a tal grado que no figuran actualmente en ningún comentario serio al ZPO.

\section{La Escuela de Rosenberg-Schwab}

La influencia de estos juristas no sólo se refleja en sus propios trabajos. A través de la influencia de su personalidad, ethos académico y la variedad de ideas estimulantes, generaron incentivos en la conformación de una escuela de discípulos consolidada. Por lo tanto, no puede ser considerado como una pura coincidencia que Peter Gottwald en 1979 como una en su Tesis de Habilitación como profesor haya prestado atención a la determinación y fijación judicial de los daños de acuerdo con $\$ 287$ ZPO. Se suma a él Reinhard Greger, en 1978 con su tesis doctoral sobre la prueba y probabilidad diciendo en relación a la obra de sus maestros: "Ambas obras se han sentido en la vanguardia de la dogmática y práctica científica y que fueron receptadas significativamente particularmente en el campo de la medida o estándar de la prueba, a partir de mi propia tesis de Habilitación sobre los interrogantes de la carga de la prueba. Puedo decir que Karl-Heinz Schwab motivó y apoyó mi labor investigativa y científica tomando como base la producción de Rosenberg a partir del año 1900, para comprobar y sopesar la coherencia de la teoría en comparación con las nuevas propuestas científicas de los años 70 del siglo pasado".

Con esa referencia a algunos trabajos sobre la prueba a partir de la influencia de Rosenberg y Schwab no podría en este momento proponer una única referencia. Más bien debo mencionar que a partir del año 1985 y fuera del círculo de discípulos de estos grandes juristas, otros han continuado las investigaciones y líneas de producción. Basta nombrar la obra de Baumgarte ${ }^{17}$ sobre la Carga de la Prueba, brillante tratado al que se le suman más de 25 publicaciones adi-

${ }^{17}$ Baumgärtel, Gottfried, Handbuch der Beweislast im Privatrecht, $1^{\text {a }}$ edición, Heymanns, Colonia, 1981. 
cionales. No puedo dejar de mencionar la importante producción e impacto de la escuela liderada por Musielak ${ }^{18}$ siguiendo la línea de investigación, especialmente por sus discípulos Christian Heinrich ${ }^{19}$ y Michael Huber ${ }^{20}$

Finalmente y pars pro toto las mencionadas obras científicas tienen finas diferencias en pequeños puntos, que no opacan la unidad en el fundamento y desarrollo a partir de la producción de Rosenberg-Schwab. A modo de ejemplo se puede tomar la obra actual del tratado sobre la Carga de la Prueba de Baumgärtel continuada por su discípulo Hans-Willi Laumen ${ }^{21}$ principalmente su segunda edición continuada que nos parece logra una -extraordinariamente- consolidada teoría como la conciliación y base para el moderno derecho probatorio. Es aquí (lo que además figura en casi todas las sugerencias prácticas) que se consigue una asombrosa uniformidad y coherencia de la libre valoración de la prueba en la interpretación del § 286 del ZPO como última ratio para el examen de los principios sobre la carga de la prueba. Se entiende el concepto de carga de la prueba como un tema central en tanto carga objetiva de la prueba (o como decía Rosenberg, la determinación de la carga de la prueba). También con gran unidad se puede entender la función de la carga de la prueba como superación del non liquet y su ámbito de aplicación en todas las normas procesales, aceptado incluso en procesos sujetos al principio inquisitivo. En particular se debe entender la distribución de la carga de la prueba como una forma de regular legalmente la distribución del riesgo que suele dar lugar a que el legislador elabore reglas de distribución para garantizar la seguridad y previsibilidad jurídica para una igual aplicación de la ley. La división de la carga de la prueba por regla se aplica en casos normales fundada en la Teoría Normativa y según las normas individuales que le sirven de fundamento. Una inversión de la carga objetiva de la prueba entonces y con la cautela que debe tenerse no puede sino ser una excepción de la regla general y con determinados requisitos lo que colabora al desarrollo del derecho no pudiendo servir un solo caso para fijar metodológicamente una disposición general. La inclusión y clasificación de la carga de la argumentación, carga subjetiva de la prueba, la verosimilitud, indicios como el juego de las presunciones dentro de este sistema se ha consolidado y logró conducir a claros resultados.

Las ambigüedades y la evolución actual del proceso judicial se da justamente en estas materias en particular donde, hasta el día de la diferenciación

\footnotetext{
${ }^{18}$ MusielaK, Hans-Joachim, Die Grundlagen der Beweislast im Zivilprozess, Duncker \& Humlot, Berlin, 1975.

${ }^{19}$ HeInRICH, Christian, Die Beweislast bei Rechtsgeschäften, Heymanns, Colonia, 1996.

${ }^{20}$ Huber, Michael, Das Beweismaß im Zivilprozess, Heymanns, Colonia, 1983.

${ }^{21}$ BaumGärTel, Gottfried-Laumen, Hanss-Willi/Prütting,Hanns, Handbuch der Beweislast, (7 volúmenes), $2^{a}$ edición, Heymanns, Colonia, 2009.
} 
entre el derecho sustantivo y la vida real, no ha logrado un desarrollo acabado. Así que no es casualidad que el concepto de distribución judicial de la carga de la prueba justamente se haya dado en los ámbitos de responsabilidad por productos en derecho del consumo, en casos de derecho médico y en los de violación de otros deberes en las profesiones liberales. Estos cambios en la carga de la prueba no deben confundirse y ser considerados como ajenos al sistema. Deben también considerarse teniendo en cuenta que en el derecho sustantivo de la responsabilidad civil hoy (y en realidad hace tiempo) el derecho judicial aún camina sobre agua tumultuosa como alguna vez lo dijo Kötz.

Por lo tanto, puede emplearse y aplicarse en la práctica aquello recurrentemente citado en el Tratado de Baumgärtel sobre la carga de la prueba y que sirven hoy de resumen sobre la discusión en torno a la prueba de los últimos 100 años. Esto incluso puede y debe resaltarse que mi predecesor en la dirección del Instituto de Derecho Procesal de la Universidad de Colonia, Gottfried Baumgärtel, casualmente también provenía de Erlangen donde habilitó como profesor bajo la dirección de, emitiendo el segundo informe o dictamen sobre su trabajo justamente Karl-Heinz Schwab.

\section{CONCLUSIONES}

Aun cuando tenemos mucho publicado en monografías y artículos científicos sobre el derecho probatorio y la carga de la prueba (sólo para mencionar la reciente obra de Maximiliano Seibl sobre la Carga de la Prueba en las normas de conflicto del año 2009), puede muchas veces llevar al olvido el fundamento sobre el que se asientan: la división entre carga objetiva y subjetiva de la prueba que permite separar y diferenciar la carga que realmente es carga de las partes (subjetiva) de la distribución normativa del riesgo probatorio (carga objetiva) establecida por el legislador en tanto instrucción a los jueces para decidir en caso de no liquet. Así se entiende que la gran división de la carga de la prueba se funda en la versión modificada de la teoría normativa y que las diversas propuestas de mejora y refinamiento son el resultado del ajuste al desarrollo del derecho sustantivo. Con mucha mayor razón y, más que nunca, estamos en materia de derecho probatorio apoyados en los hombros Leo Rosenberg y Karl-Heinz Schwab. Esto incluye también por cierto a su círculo de discípulos. Así el estado actual del derecho de la prueba muestra claramente que la ley puede lograr un gran avance y es una verdadera ciencia. El derecho de la prueba ha creado e identificado los principios básicos como las normas sobre la carga de la prueba, que no pueden ser eliminados por el legislador. Más bien se demuestra aquí que en todo sistema procesal deben diferenciarse y separarse en forma estricta la libre apreciación de la prueba, el nivel, medida o estándar de la prueba y finalmente los fundamentos de la carga de la prueba. 
Las normas jurídicas individuales, y por lo tanto la distribución del riesgo entre las partes, pueden ser controlados por el legislador en cada caso concreto de acuerdo a una aplicación e interpretación teleológica del derecho sustantivo. Pero incluso en esos casos debe construirse todo a partir de los fundamentos presentados en el moderno derecho probatorio. Los esfuerzos en materia de prueba emprendidos como aventura individual y solitaria del legislador o incluso de un juez no resultan admisibles.

Karl-Heinz Schwab siempre ha entendido, y en ello fue muy consciente como discípulo de Leo Rosenberg, que debía actuar con previsión y precaución. Uno comprenderá que ello también obliga a este humilde discípulo de Karl-Heinz Schwab a comprometerse con este trabajo, y recordar con gratitud a su maestro. 